\title{
Application of type Timoshenko equations for shock action
}

\author{
Evgeniy Zveryaev ${ }^{1, *}$, Evgeniy Larionov ${ }^{2}$ \\ ${ }^{1}$ Keldysh Institute of Applied Mathematics, Miusskaya sq. 4, Moscow, 125047, Russia \\ ${ }^{2}$ Moscow state university of civil engineering, Yaroslavskoye shosse, 26, Moscow, Russia, 129337
}

\begin{abstract}
The generalized Timoshenko type equations are obtained from the plane problem equations of the theory elasticity. They might be applied for quick acting forces or displacements problems as the transversal shock on a beam or quick displacement of beam ends. The quick and slow solutions components are extracted. The quick ones describe the propagation of the shear wave due to the action of a concentrated force or an explosion; the slow solutions are generated by the common verification of the boundary conditions the slow and quick solutions.
\end{abstract}

\section{Introduction}

Under the notion of quick action we understand the quick varying action as a transversal shock on the beam with a force, an explosion near the height building considering as a beam of complex structure, or a moving of the ground resulting earthquake etc. It is believed the solution of such problems. It is believed, the solution of such problems should be made taking into account the wave character of the solution in the impacted body. In the theory of strength of materials it is proposed for these purposes to use the Timoshenko equation (1922) for a beam [1] and equations of the Timoshenko type, taking into account the inertia of the rotation and shear of the element, for a plate or shell. The procedure to find the wave solution of these equations reduces to apply the step load to the infinite body and the equations of state undergo a Laplace transformation with respect to the time and Fourier transformation with respect to coordinates. The inverse transformation is made numerically. It must be said Timoshenko's equation is extremely difficult to solve, and the author does not know the available understandings of either numerical or analytical solutions. The derivation and analysis of the equations of the theory of elasticity, given in this paper, showed that under rapid influences on the strip, two main types of motion are distinguished in it: longitudinal shear wave and transverse vibrational (not wave) lowfrequency (classical).

In order to understand the behavior of the solution of the Timoshenko equation [2]

$$
E I \frac{\partial^{4} y^{*}}{\partial x^{* 4}}-\rho I\left(1+\frac{E}{k^{\prime} G}\right) \frac{\partial^{4} y^{*}}{\partial x^{* 2} \partial t^{* 2}}+\frac{\rho^{2} I}{k^{\prime} G} \frac{\partial^{4} y^{*}}{\partial t^{* 4}}+\rho F \frac{\partial^{2} y^{*}}{\partial t^{* 2}}=0
$$

\footnotetext{
* Corresponding author: zveriaev@gmail.com
} 
where $E$ is the Young's modulus, $G$ is the shear modulus, $k^{\prime}$ is the coefficient depending on the shape of the cross section, $F$ is the area of the cross section, $\rho$ is the mass of the beam length unit, $y^{*}$ is the deflection, $x^{*}$ is the beam axis coordinate, $t^{*}$ is the time, $I$ is the moment of inertia of the cross section. The sign ${ }^{*}$ marks the dimensional values.

There are various recommendations for choosing the value of the coefficient $k^{\prime}$ : for a rectangular cross-section the value 0.833 [2] is used, the value $2 / 3$ and value $3 / 4$ are for the circular section [3]; it was pointed out $\pi^{2} / 12$, but values $5 / 6$ and $5 /(6-v)$ were also allowed [4].

If we put the time differentiation operator in equation (1) equal to zero, Timoshenko's equation turns into the classical bending equation of a beam. This means that the intuitive theory of Timoshenko exists only for dynamic problems. However, the constructive derivation of the equations on the basis of the mapping contraction principle from the equations of the theory of elasticity yields a static version of the Timoshenko type equations $[5,6]$. The aim of this paper is to establish the additional equations to the classical ones taking into account the fast phenomena corresponding to the concentrated force in space and time.

\section{Equations of static bending}

We assign a long rectangular strip to a rectangular coordinate system $x^{*}, z^{*}$, so that $0 \leq x^{*} \leq l,-h \leq z^{*} \leq h$, where $2 h$ is the thickness, $l$ is the longer of the strip. The strip bends under the action of the load $q^{*}=q^{*}(x)$ distributed on the top edge and directed downwards. We introduce dimensionless coordinates, $x=x^{*} / l, z=z^{*} / h$ dimensionless displacements $u=u^{*} / h, w=w^{*} / h$ along the axes $x, z$ respectively, and dimensionless stresses $\sigma_{x}=\sigma_{x}^{*} / E, \sigma_{z}=\sigma_{z}^{*} / E, \tau=\tau^{*} / E$. The equations of the plan problem of theory of elasticity

$$
\begin{gathered}
\frac{\partial \sigma_{z}^{*}}{\partial z^{*}}+\frac{\partial \tau^{*}}{\partial x^{*}}=0, \quad \frac{\partial \sigma_{x}^{*}}{\partial x^{*}}+\frac{\partial \tau^{*}}{\partial z^{*}}=0 \\
\sigma_{x}^{*}=\frac{E}{1-v^{2}}\left(\varepsilon_{x}+v \varepsilon_{z}\right), \quad \tau^{*}=\frac{E}{2(1+v)} \gamma, \quad \sigma_{z}^{*}=\frac{E}{1-v^{2}}\left(\varepsilon_{z}+v \varepsilon_{x}\right) \\
\varepsilon_{z}=\frac{\partial w^{*}}{\partial z^{*}}, \quad \varepsilon_{x}=\frac{\partial u^{*}}{\partial x^{*}}, \quad \gamma=\frac{\partial u^{*}}{\partial z^{*}}+\frac{\partial w^{*}}{\partial x^{*}}
\end{gathered}
$$

describing the stress-strain state of the strip we reduce with an identical transformations to the form allowing us to calculate all searching unknowns when the quantities $w, \tau$ are known

$$
\begin{aligned}
& \frac{\partial u}{\partial z}=-\varepsilon \frac{\partial w}{\partial x}+2(1+v) \tau, \quad \frac{\partial \sigma_{z}}{\partial z}=-\varepsilon \frac{\partial \tau}{\partial x}, \quad \varepsilon_{x}=\varepsilon \frac{\partial u}{\partial x} \\
& \sigma_{x}=\varepsilon_{x}+v \sigma_{z}, \quad \varepsilon_{z}=\left(1-v^{2}\right) \sigma_{z}-v \varepsilon_{x}, \quad \frac{\partial w}{\partial z}=\varepsilon_{z}, \quad \frac{\partial \tau}{\partial z}=-\varepsilon \frac{\partial \sigma_{x}}{\partial x}
\end{aligned}
$$

Here $\varepsilon=h / l$ is smoll parametr.

In fact from the first two equations when $w, \tau$ are known, we calcul $u, \sigma_{z}$ and via $u$ calcul $\varepsilon_{x}$. The relations of elasticity permit to calcul $\sigma_{x}, \varepsilon_{z}$. The last two equations again give the same values $w, \tau$. This fact in the functional analyses is treated as the Banach fixed-point theorem (also known as the contraction mapping theorem or contraction 
mapping principle). Usually the exact values of the quantities $w, \tau$ are unknown. Therefore, we can choose the approximate quantities $w_{0}, \tau_{0}$ in the first two equations and calculate in the last two $w_{1}, \tau_{1}$ as the values of the first approximation. The process is treated as a method of simple iterations (the Banach theorem, 1922). The presence of the small parameter $\varepsilon=h / l$ makes it possible to compare the method of the simple iterations with the method of asymptotic integration and to estimate the conditions for asymptotic convergence.

We give the values of the initial approximation $w=w_{0}(x), \tau=\tau_{0}(x)$ as a function of only one coordinate $x$. This means that, in the zeroth approximation, there is no change in the thickness of the strip and a uniform distribution of the tangential stress that is usually used as a hypothesis in the derivation of the equations of the plates and shells theories. In the first approximation, a correction is calculated for the transverse compression and distribution of the tangential stress.

Calculating the integrals of the system (2) by the described method, we write the boundary conditions on the long sides of the strip

$$
\tau=\sigma_{z}=0 \text { при } z=-1, \tau=0, \sigma_{z}=-q \text { при } z=1 ; \quad\left(q=q^{*} / E\right)
$$

We shall use them as epy first-approximation quantities

$$
\begin{aligned}
& \tau=\left[\varepsilon^{3} w_{0}^{\prime \prime \prime}-(2+v) \varepsilon^{2} \tau_{0}^{\prime \prime}\right] \frac{z^{2}}{2}+\tau_{0}-\left(\varepsilon^{2} u_{0}^{\prime \prime}+v \varepsilon \sigma_{z 0}^{\prime}\right) z \\
& \sigma_{z}=-\left[\varepsilon^{4} w_{0}^{\prime \prime \prime \prime}-(2+v) \varepsilon^{3} \tau_{0}^{\prime \prime \prime}\right] \frac{z^{3}}{6}-\varepsilon \tau_{0}^{\prime} z+\left(\varepsilon^{3} u_{0}^{\prime \prime \prime}+v \varepsilon^{2} \sigma_{z 0}^{\prime \prime}\right) \frac{z^{2}}{2}+\sigma_{z 0}
\end{aligned}
$$

Here and below, the prime denotes the differentiation with respect to $x$.

After substituting the stresses into the boundary conditions, we obtain the four equations for the determination of the functions $w_{0}, \tau_{0}, u_{0}, \sigma_{z 0}$

$$
\begin{gathered}
\varepsilon^{2} u_{0}^{\prime \prime}+v \varepsilon \sigma_{z 0}^{\prime}=0 \\
\varepsilon^{3} u_{0}^{\prime \prime \prime}+v \varepsilon^{2} \sigma_{z 0}^{\prime \prime}+2 \sigma_{z 0}=-q \\
\varepsilon^{3} w_{0}^{\prime \prime \prime}-(2+v) \varepsilon^{2} \tau_{0}^{\prime \prime}+2 \tau_{0}=0, \\
\varepsilon^{4} w_{0}^{\prime \prime \prime}-(2+v) \varepsilon^{3} \tau_{0}^{\prime \prime \prime}+6 \varepsilon \tau_{0}^{\prime}=3 q
\end{gathered}
$$

The first two equations refer to the tension-compression, the second two to the bending, and we will be interested only in the last one. We present the searched unknowns and load in the form of the sum of rapidly varying components along $x$ and slowly varying components, i.e.

$$
w_{0}=w_{0}^{s}+w_{0}^{q}, \tau_{0}=\tau_{0}^{s}+\tau_{0}^{q}, q=q^{s}+q^{q}
$$

Substituting them into the first system and demanding that the slow and fast component go to zero separately in each equation, we obtain equations

$$
\begin{gathered}
\varepsilon^{3} w_{0}^{s \prime \prime \prime}+2 \tau_{0}^{s}=0 \\
\varepsilon^{4} w_{0}^{s^{\prime \prime \prime \prime}}+6 \varepsilon \tau_{0}^{s^{\prime}}=3 q^{s} \\
\varepsilon^{3} w_{0}^{q \prime \prime \prime}-(2+v) \varepsilon^{2} \tau_{0}^{q \prime \prime}+2 \tau_{0}^{q}=0, \\
\varepsilon^{4} w_{0}^{q \prime \prime \prime}-(2+v) \varepsilon^{3} \tau_{0}^{q \prime \prime \prime}+6 \varepsilon \tau_{0}^{q \prime}=3 q^{q}
\end{gathered}
$$

In the first two equations, the terms with the second and third derivatives of $\tau_{0}^{s}$ are rejected as small compared with the principal $\tau_{0}^{q}$ and $\tau_{0}^{q^{\prime}}$, respectively. Excluding $\tau_{0}^{s}$ from the 
second equation with the help of the first one we obtain the classical equation of bending and an additional equation for determining the tangential stress.

$$
\frac{2}{3} \varepsilon^{4} w_{0}^{s^{\prime \prime \prime \prime}}=-q, \quad \varepsilon \tau_{0}^{s^{\prime}}=\frac{3}{4} q
$$

The second system of equations is transformed to the form

$$
\begin{aligned}
& \frac{2}{3} \varepsilon^{4} w_{0}^{q{ }^{\prime \prime \prime}}=-q^{q}+\frac{\varepsilon^{2}}{k^{2}} q^{q^{\prime \prime}} \\
& \frac{\varepsilon^{2}}{k^{2}} \tau_{0}^{q^{\prime \prime}}-\tau_{0}^{q}=\frac{1}{2} \varepsilon^{3} k^{2} w_{0}^{q \prime \prime \prime}
\end{aligned}
$$

The two systems describe the action of the slowly and rapidly changing loads on a strip (beam) and can be declared in correspondence with equation (1) as a static version of the theory of Timoshenko's type. Having determined from this $w_{0}^{s}, w_{0}^{q}, \tau_{0}^{s}, \tau_{0}^{q}$ we obtain by means of the formulas (4) the stresses $\tau, \sigma_{z}$. Formulas for the remaining searched unknowns can be found in $[4,5]$.

If in the first equation of the system (5) the load is chosen so that it satisfies the equation $-q^{q}+\frac{\varepsilon^{2}}{k^{2}} q^{q^{\prime \prime}}=0$, and this will be, for example, the function

$$
q^{q}=\left\{\begin{array}{lll}
B_{1} e^{k(x-b) / \varepsilon} & \text { при } x \leq b \\
B_{2} e^{-k(x-b) / \varepsilon} & \text { при } x \geq b
\end{array}\right.
$$

and choose the constants $B_{1}=B_{2}=P k \varepsilon^{-1} / 2(P=$ const $)$, we see that

$$
\lim _{\varepsilon \rightarrow 0} q^{q}=P \delta(x-b)
$$

The value $P$ determines the intensity of the applied force corresponding to the local load $q^{q}$. In this case, the first equation of the system (5) becomes homogeneous and does not have any solution among the quickly varying functions, hence $w_{0}^{q}=0$. Accordingly, the second equation also becomes homogeneous and has a boundary layer type solution.

\section{Dynamic equations for strip}

We apply the procedure described above for constructing the equations of motion for a long strip. Adding dimensionless inertial terms to the static bending equations (2), we obtain the following equations of motion

$$
\begin{aligned}
& \frac{\partial u}{\partial z}=-\varepsilon \frac{\partial w}{\partial x}+2(1+v) \tau, \quad \frac{\partial \sigma_{z}}{\partial z}=-\varepsilon \frac{\partial \tau}{\partial x}+m^{2} \ddot{w} \\
& \sigma_{x}=\varepsilon \frac{\partial u}{\partial x}+v \sigma_{z}, \quad \varepsilon_{z}=-v \varepsilon \frac{\partial u}{\partial x}+\left(1-v^{2}\right) \sigma_{z}, \quad\left(m^{2}=\frac{\rho h^{2}}{E T^{2}}\right) \\
& \frac{\partial w}{\partial z}=\varepsilon_{z}, \quad \frac{\partial \tau}{\partial z}=-\varepsilon \frac{\partial \sigma_{x}}{\partial x}+m^{2} \ddot{u}
\end{aligned}
$$

where $\rho$ is the specific density of the strip material, $t=t^{*} / T$ is dimensionless time ( $t^{*}$ is dimensional time), $T$ is some characteristic period of oscillation of a strip (beam), having length $l$ and height $2 h$. The dot over a letter means the differentiation with respect to $t$.

Here, as well as in the static problem, it is possible consequentially calculate all the unknowns, assuming $w, \tau$ known. To find the approximate solution by the simple iteration method, we can choose the initial approximation and do the calculations according to the scheme 


$$
w_{0}, \tau_{0} \Rightarrow u_{0}, \sigma_{z 0} \Rightarrow \varepsilon_{x 0}, \sigma_{x 0}, \varepsilon_{z 0} \Rightarrow w_{1}, \tau_{1}
$$

To show the applicability of the simple iterations method to the integration of the system (6), we transform it

$$
\begin{array}{ll}
\frac{\partial u}{\partial z}=-\varepsilon \frac{\partial w}{\partial x}+2(1+v) \tau, & \frac{\partial \sigma_{z}}{\partial z}=m^{2} \ddot{w}-\varepsilon \frac{\partial \tau}{\partial x} \\
\frac{\partial w}{\partial z}=-v \varepsilon \frac{\partial u}{\partial x}+\left(1-v^{2}\right) \sigma_{z}, & \frac{\partial \tau}{\partial z}=-\varepsilon^{2} \frac{\partial^{2} u}{\partial x^{2}}+m^{2} \ddot{u}-v \varepsilon \frac{\partial \sigma_{z}}{\partial x}
\end{array}
$$

and rewrite it in vector form. We introduce vectors $\mathbf{u}=\left\{u, \sigma_{z}\right\}, \mathbf{w}=\{w, \tau\}$ and matrix operators

$$
\mathbf{A}_{1}=\left\|\begin{array}{cc}
-\varepsilon \frac{\partial}{\partial x} & 2(1+v) \\
m^{2} \frac{\partial^{2}}{\partial t^{2}} & -\varepsilon \frac{\partial}{\partial x}
\end{array}\right\|, \quad \mathbf{A}_{2}=\left\|\begin{array}{cc}
-v \varepsilon \frac{\partial}{\partial x} & 1-v^{2} \\
-\varepsilon^{2} \frac{\partial^{2}}{\partial x^{2}}+m^{2} \frac{\partial^{2}}{\partial t^{2}} & -v \varepsilon \frac{\partial}{\partial x}
\end{array}\right\|
$$

With them the system is written as follows

$$
\frac{\partial \mathbf{u}}{\partial z}=\mathbf{A}_{1} \mathbf{w}, \quad \frac{\partial \mathbf{w}}{\partial z}=\mathbf{A}_{2} \mathbf{u}
$$

Integrating these equations with respect to $z$, we reduce them to a single equation

$$
\mathbf{w}=\int \mathbf{A}_{2}\left(\int \mathbf{A}_{1} \mathbf{w} d z+C\right) d z+D
$$

where $C, D$ are the arbitrariness of integration, which are functions of the coordinate $x$. The solution of the equation can be searched by the simple iteration method

$$
\mathbf{w}_{(n+1)}=\int \mathbf{A}_{2}\left(\int \mathbf{A}_{1} \mathbf{w}_{(n)} d z+C\right) d z+D
$$

where $n$ is number of the iteration.

Thus, the formal feature of the applicability of the simple iteration method is fulfilled.

Performing the calculations according to scheme (7) and writing down the expressions for all the searched unknowns

$$
\begin{aligned}
& u=-\varepsilon w_{0}^{\prime} z+2(1+v) \tau_{0} z, \\
& w=w_{0}+\left[\left(1-v^{2}\right) \varepsilon^{2} m^{2} \ddot{w}_{0}+v \varepsilon^{2} w_{0}^{\prime \prime}\right] \frac{z^{2}}{2}-(1+v)^{2} \varepsilon \tau_{0}^{\tau_{0}} \frac{z^{2}}{2}, \\
& \sigma_{x}=\left(-\varepsilon^{2} w_{0}^{\prime \prime}+v \varepsilon^{2} m^{2} \ddot{w}_{0}\right) z+(2+v) \varepsilon \tau_{0}^{\prime} z, \\
& \tau=\tau_{0}+\left[\varepsilon^{3} w_{0}^{\prime \prime \prime}-(1+v) \varepsilon m^{2} \ddot{w}_{0}^{\prime}-(2+v) \varepsilon^{2} \tau_{0}^{\prime \prime}+2(1+v) m^{2} \ddot{\tau}_{0}\right] \frac{z^{2}}{2}, \\
& \sigma_{z}=-\left[\varepsilon^{4} w_{0}^{\prime \prime \prime}-(1+v) \varepsilon^{2} m^{2} \ddot{w}_{0}^{\prime \prime}-(2+v) \varepsilon^{3} \tau_{0}^{\prime \prime \prime}+2(1+v) \varepsilon m^{2} \ddot{\tau}_{0}^{\prime}\right] \frac{z^{3}}{6}-\varepsilon \tau_{0}^{\prime} z+m^{2} \ddot{w}_{0} z,
\end{aligned}
$$

we use stresses $\tau, \sigma_{z}$ to satisfy the boundary conditions (3). We obtain equations for the definition of functions $w_{0}, \tau_{0}$.

$$
\begin{aligned}
& \varepsilon^{3} w_{0}^{\prime \prime \prime}-(1+v) \varepsilon m^{2} \ddot{w}_{0}^{\prime}-(2+v) \varepsilon^{2} \tau_{0}^{\prime \prime}+2(1+v) m^{2} \ddot{\tau}_{0}+2 \tau_{0}=0 \\
& \varepsilon^{4} w_{0}^{\prime \prime \prime}-(1+v) \varepsilon^{2} m^{2} \ddot{w}_{0}^{\prime \prime}-(2+v) \varepsilon^{3} \tau_{0}^{\prime \prime \prime}+2(1+v) \varepsilon m^{2} \ddot{\tau}_{0}^{\prime}+6 \varepsilon \tau_{0}^{\prime}-6 m^{2} \ddot{w}_{0}=3 q
\end{aligned}
$$

From the second equation we subtract the first one, multiplied by $\varepsilon$ and differentiated with respect to $x$. Thus we obtain the relation

$$
\varepsilon \tau_{0}^{\prime}=3\left(2 m^{2} \ddot{w}_{0}+q\right) / 4
$$

Eliminating $\tau_{0}$ from the second equation with help of this equation, we obtain the equation 


$$
\begin{aligned}
& \frac{2}{3}\left[\varepsilon^{4} w_{0}^{\prime \prime \prime}-\left(4+\frac{5}{2} v\right) \varepsilon^{2} m^{2} \ddot{w}_{0}^{\prime \prime}+3(1+v) m^{4} \dddot{w}_{0}\right]+2 m^{2} \ddot{w}_{0}= \\
& =-q+\frac{2+v}{2} \varepsilon^{2} q^{\prime \prime}-(1+v) m^{2} \ddot{q}
\end{aligned}
$$

similar to the left-hand side of the equation of Tymoshenko (1) reduced to the dimensionless form,

$$
\frac{2}{3}\left[\varepsilon^{4} y^{\prime \prime \prime}-\left(1+\frac{2(1+v)}{k^{\prime}}\right) \varepsilon^{2} m^{2} \ddot{y}^{\prime \prime}+\frac{2(1+v)}{k^{\prime}} m^{4} \dddot{y}\right]+2 m^{2} \ddot{y}=0
$$

where, in addition to the previously introduced dimensionless quantities, the dimensional deflection was replaced by dimensionless $y^{*}=h y$. In the coefficients there is an empirical value $k^{\prime}$ and substitutions $I=2 / 3 b h^{3}, F=2 b h$ ( $b$ is the width of the beam section) are made. When $k^{\prime}=2 / 3$ this equation is close to the equation (10)

$$
\frac{2}{3}\left[\varepsilon^{4} y^{\prime \prime \prime}-(4+3 v) \varepsilon^{2} m^{2} \ddot{y}^{\prime \prime}+3(1+v) m^{4} \dddot{y}\right]+2 m^{2} \ddot{y}=0
$$

Thus, the Timoshenko equation (11) obtained from the system of two equations (9), we shall call it so, should be supplemented by some second equation from the same system or a combination of them. We differentiate the first equation of the system (9) with respect $x$, multiply it by $\varepsilon$ and add it with the second. It gives

$$
2 \varepsilon^{4} w_{0}^{\prime \prime \prime}-2(1+v) \varepsilon^{2} m^{2} \ddot{w}_{0}^{\prime \prime}-2(2+v) \varepsilon^{3} \tau_{0}^{\prime \prime \prime}+4(1+v) \varepsilon m^{2} \ddot{\tau}_{0}^{\prime}+8 \varepsilon \tau_{0}^{\prime}+6 m^{2} \ddot{w}_{0}=3 q
$$

Using the relation (10) in the form $2 m^{2} \ddot{w}_{0}=\frac{4}{3} \varepsilon \tau_{0}^{\prime}-q$, we exclude $\ddot{w}_{0}$ from the second term. After reduction of such terms, we obtain the equation

$$
\left[\frac{2}{3} \varepsilon^{4} w_{0}^{\prime \prime \prime}+2 m^{2} \ddot{w}_{0}\right]+\varepsilon \frac{\partial}{\partial x}\left[-\frac{16+10 v}{9} \varepsilon^{2} \tau_{0}^{\prime \prime}+\frac{4}{3}(1+v) m^{2} \ddot{\tau}_{0}+\frac{8}{3} \tau_{0}\right]=q-\frac{1+v}{3} \varepsilon^{2} q^{\prime \prime}
$$

Here the first term in square brackets describes the transverse movement of the rod, the second describes longitudinal shear movement.

We add estimates of the inertial terms to these equations. To do this, we must select the unit of time in equations (6). Let us write out the known equations of longitudinal and transverse oscillations of a rod of rectangular cross section, having written them down in a dimensionless form

$$
\varepsilon^{2} u^{\prime \prime}-m_{l}^{2} \ddot{u}=0, \frac{2}{3} \varepsilon^{4} y^{\prime \prime \prime}-m_{t}^{2} \ddot{y}=0
$$

where the notations are introduced $m_{l}^{2}=\frac{\rho h^{2}}{E T_{l}^{2}}, m_{t}^{2}=\frac{\rho h^{2}}{E T_{t}^{2}}, u$ is the longitudinal displacement of the points of the rod, $y$ is the transversal displacements of the neutral axis. Understanding under $T_{l}$ the characteristic period of the longitudinal vibrations of the rod and $T_{t}$ the characteristic period of the transverse vibrations, we conclude $m_{l}^{2} \sim \varepsilon^{2}$ and $m_{t}^{2} \sim \varepsilon^{4}$. In accordance with these estimates, we make the substitution $m_{l}=\varepsilon n_{l}, m_{t}=\varepsilon^{2} n_{t}$ in equation (12), setting $n_{l}, n_{t} \sim \varepsilon^{0}$

$$
\varepsilon^{4}\left[\frac{2}{3} w_{0}^{\prime \prime \prime}+2 n_{t}^{2} \ddot{w}_{0}\right]+\varepsilon \frac{\partial}{\partial x}\left[-\frac{16+10 v}{9} \varepsilon^{2} \tau_{0}^{\prime \prime}+\frac{4}{3}(1+v) \varepsilon^{2} n_{l}^{2} \ddot{\tau}_{0}+\frac{8}{3} \tau_{0}\right]=q-\frac{1+v}{3} \varepsilon^{2} q^{\prime \prime}
$$

If the load on the right part is rapidly varying with respect to $x$, the particular solution is determined from the equation 


$$
\varepsilon \frac{\partial}{\partial x}\left[-\frac{16+10 v}{9} \varepsilon^{2} \tau_{0}^{\prime \prime}+\frac{4}{3}(1+v) \varepsilon^{2} n_{l}^{2} \ddot{\tau}_{0}+\frac{8}{3} \tau_{0}\right]=q-\frac{1+v}{3} \varepsilon^{2} q^{\prime \prime}
$$

or when $q$ is a slowly changing load it determined from the equation

$$
\varepsilon^{4}\left[\frac{2}{3} w_{0}^{\prime \prime \prime}+2 n_{t}^{2} \ddot{w}_{0}\right]=q
$$

The general solution is determined from the equations

$$
\frac{2}{3} w_{0}^{\prime \prime \prime}+2 n_{t}^{2} \ddot{w}_{0}=0, \quad \varepsilon \frac{\partial}{\partial x}\left[-\frac{8+5 v}{3} \varepsilon^{2} \tau_{0}^{\prime \prime}+2(1+v) \varepsilon^{2} n_{l}^{2} \ddot{\tau}_{0}+4 \tau_{0}\right]=0
$$

The first equation gives a slowly varying integral with respect to $x$, the second one gives the rapidly varying integral, depending on the argument $x / \varepsilon$, having wave properties.

\section{Conclusion}

So, the main conclusion that can be drawn from the present paper is the content in the wellknown Timoshenko equation of two types of main motions, the high-speed shear wave and low-frequency transverse oscillations. They should be selected from the equations and integrated separately, regardless of the type of solution, analytically or numerical simulation.

\section{References}

1. S. Timoshenko, Phil. Mag. 43, 253 (1922)

2. S. Timoshenko, D.H. Young, W. Weaver, Vibration Problems in Engineering (N.Y.etc. : Wiley, 1974)

3. Ya. S. Uflyand, Prikl Mat Mekh, 12, 3 (1948)

4. P. A. Zhilin, T.P. Il'icheva, J Appl Mech Tech Phy, 25, 1, (1984)

5. Ye. Zveryayev, G.I. Makarov, Prikl Mat Mekh, 72, 2 (2008)

6. Ye. Zveryayev, Prikl Mat Mekh, 67, 3 (2003) 